\title{
A new disease with virus of citrus fruits in the Middle East ${ }^{1}$
}

In January 1957, Henri Chapot, visiting Lebanon, Syria and Turkey, discovers the very clear symptoms of "Stubborn", a citrus tree virus disease then little known. Here what it reports:

"In the United States, the disease in question is called "Stubborn" or disease of the stubbornness. It is supposed to be caused by a virus that Fawcett named Citrivir pertinaciae, i.e., virus of the citrus stubbornness.

The "Stubborn" was discovered in 1929 in California... For a long time it was regarded as a curiosity...

Meanwhile, this disease had been discovered by Bitancourt in Brazil in its form of "crazy signal"... For our part, we recognized this disease in North Africa since 1949... In January 1956, we discovered it into two or three rather distant points in French Guinea, with undoubtful symptoms...

The visit of the citrus areas of Lebanon was followed by a visit of corresponding zones of Syria and then of Turkish Mediterranean coasts.

Lebanon: In the South of Tyr, one of the most beautiful orange plantations of Lebanon is highly contaminated... Between Tyr and Saïda, a 6-years old plantation of orange trees "Valencia Late" is affected by generalized symptoms... same situation in the very Saïda... and in the North of Lebanon.

Syria: In Syria, two areas were prospected, that of Tartous and that of Lattakié... The "Stubborn" was recognized in various orange orchards and on various varieties.

Turkey: In the nursery station of Dortyöl... the existence of the "Stubborn" is absolutely undoubtly ... From the Iskenderoun region, we went in that of Içel (Mersine). In the centre of a very beautiful plantation, we located old trees relatively infected by "Stubborn".

In conclusion, we will point out that:

- the symptoms observed in the three countries, Lebanon, Syria and Turkey, are extremely typical and hardly allow to accuse another disease but the "Stubborn";

- the increasingly frequent discovery of trees from local varieties, often old, variously affected by "Stubborn", makes think of an infection by an animal vector;

- the grafts introduced from Palestine, in particular those of "Washington Navel" and "Valencia Late", gave all, later on, sick trees. As this disease was given with many chances of reason as being the "Stubborn", as, in addition, this disease was not announced under this name in Palestine whereas the "Little leaf disease" and the "Xeromorphosis" were well known there, we do not hesitate to regard these three names as absolutely synonymous;

- the case of Calabrian "Oval" orange and that of the common mandarin tree will require additional studies."

\footnotetext{
${ }^{1}$ See Fruits 12 (1) 3-7.
} 


\section{Lu dans Fruits, il y a 50 ans...}

\section{Une nouvelle maladie à virus des agrumes dans le Moyen-Orient ${ }^{1}$}

En janvier 1957, Henri Chapot en visite au Liban, en Syrie et en Turquie, découvre les symptômes très nets du "stubborn ", virose des agrumes alors peu connue. Voici ce qu'il rapporte :

"La maladie dont il est question est appelée aux États-Unis "Stubborn" ou maladie de l'entêtement. On l'attribue à un virus que Fawcett nomma Citrivir pertinaciae, c'est-à-dire virus de l'entêtement des agrumes.

Le "Stubborn" fut découvert en 1929 en Californie...Pendant longtemps, elle fut considérée comme une curiosité...

Entre temps, cette maladie avait été découverte par Bitancourt au Brésil sous sa forme de " crazy top "...Pour notre part, nous avons reconnu cette maladie en Afrique du Nord depuis 1949...En janvier 1956, nous l'avons découverte en deux ou trois points assez éloignés entre eux de la Guinée française et avec des manifestations qui ne laissaient aucun doute...

À la visite des régions agrumicoles du Liban succéda celles des zones correspondantes de Syrie, puis des côtes méditerranéennes turques.

Liban : Au Sud de Tyr, une des plus belles plantations d'orangers du Liban est atteinte à un très haut degré... Entre Tyr et Saïda, une plantation d'orangers "Valencia Late " âgée de six ans environ présente des symptômes généralisés... À Saïda même, une vieille orangeraie présentait des déformations de fruits typiques du "stubborn "... De même au Nord du Liban.

Syrie : En Syrie, deux régions furent prospectées, celle de Tartous et celle de Lattakié...Le "stubborn " a été reconnu dans diverses orangeraies et sur diverses variétés.

Turquie : À La Station-pépinière de Dortyöl, l'existence du "stubborn " ne fait absolument aucun doute...De la région d'Iskenderoun, nous nous rendîmes dans celle d'Içel (Mersine). Au milieu d'une très belle plantation, nous repérâmes des arbres relativement âgés atteints de "stubborn "...

En conclusion, nous ferons remarquer que :

- les symptômes observés dans les trois pays, Liban, Syrie et Turquie, sont extrêmement caractéristiques et ne permettent guère d'incriminer une autre maladie que le "stubborn ";

- la découverte de plus en plus fréquente d'arbres de variétés locales, souvent âgés, atteints à des degrés divers du "stubborn ", fait songer à une infection par un vecteur animal ;

- les greffons introduits de Palestine, en particulier ceux de "Washington Navel " et de "Valencia Late ", ont tous donné ultérieurement des arbres malades. Comme cette maladie a été déterminée avec beaucoup de chances de raison comme étant le "stubborn ", comme d'autre part cette maladie n'a pas été signalée sous ce nom en Palestine alors que la "Little leaf disease " et le "Xeromorphosis" y étaient bien connus, nous n'hésitons pas à considérer ces trois noms comme absolument synonymes ;

- le cas de l'orange "Ovale calabraise " et celui du mandarinier commun demanderont des études supplémentaires."

Jacky Ganry

\footnotetext{
${ }^{1}$ Voir Fruits 12 (1) 3-7.
} 